\title{
Meningioma Associated with Brain Aneurysm: Report of Two Cases
}

\author{
Sayoa Alvarez de EULATE-BERAMENDI, Marco Antonio ALVAREZ-VEGA, Julio Cesar GUTIERREZ-MORALES, \\ Antonio LOPEZ-GARCIA
}

Hospital Universitario Central de Asturias, Department of Neurosurgery, Oviedo/Asturias, Spain

\section{ABSTRACT}

The occurrence of brain tumors together with aneurysms has been considered as an uncommon phenomenon. However, its incidence may be underestimated. This coexistence is a diagnostic challenge, but also a therapeutic one as no consensus has been reached. We report two cases of a 71 and 67 years old patient with a meningioma and aneurysm: one noticed and treated before with good outcome and the other without treatment before surgery with fatal outcome. The different outcome of these patients shows the importance of vascular study in surgical planning. Treatments options are changing and although some authors think the pathology that causes symptoms should be treated first, endovascular treatment of the aneurysm is a safe option to prevent aneurysm rupture during surgery.

KEYWORDS: Aneurysm, Meningioma, Coincidence

\section{INTRODUCTION}

$\mathrm{T}$

The occurrence of brain tumors together with aneurysms has been considered as an uncommon phenomenon.

However, its incidence may be underestimated. With the increased use of preoperative tumor embolization, aneurysm may be identified more frequently. Although aneurysm may coexist with different types of brain tumors as pituitary adenoma, glioma, lipoma or metastatic tumor $(6,11,12,15)$, brain tumor which is most commonly associated with an aneurysm is meningioma (8). This coexistence is a diagnostic challenge, but also a therapeutic one as no consensus has been reached. We report two cases of meningioma and aneurysm with preoperative vascular study, to show the importance of considering underlying vascular abnormalities when resecting tumors raising questions of surgical planning.

\section{CASE REPORTS}

\section{CASE 1}

A 71-year-old female patient, with hypertension and insulin- dependent diabetes, was suffering from gait disturbance for several months. She was admitted to another hospital and a computed tomography (CT) and magnetic resonance (MR) revealed an extra-axial mass in left posterior fossa with tentorium implantation. The most likely diagnosis was a meningioma and she was admitted at the department of neurosurgery. A preoperative cerebral angiography was performed for the purpose of supra-selective embolisation of neoplastic vessels. Rather, a right Posterior Communicating Artery Aneurysm was found (Figure 1A, B). On the next day, with the patient's consent, an endovascular embolisation of the aneurysm was performed. Four days after the patient underwent suboccipital craniotomy to remove the tumor. Histopathological study of the tumor revealed a meningothelial meningioma. The patient's condition improved postoperatively with mild dysmetria so she received rehabilitation treatment and was discharged home.

\section{CASE 2}

A 67-year-old female patient, with a history of ulcerative colitis, presented with confusion and behavior disturbance. She was 
studied by neurologist who found a left temporal mass in CT scan, with surrounding oedema and mass effect. She was admitted to our department and a MR confirmed a left medial sphenoid wing meningioma. CT-angiography was performed to analyse the vessels localization near paraclinoid portion. No aneurysms were found. There were no hypertension or ventilation problems during anesthetic induction. A pterional craniotomy was performed to remove the tumor, however, while removing the bone flap, untreatable cerebral oedema was found. An immediate CT scan showed subarachnoid haemorrhage and a right paraclinoid pseudoaneurysm (Figure 2A-C). Despite coiling the aneurysm and doing a decompressive craniotomy the patient died 2 days after the surgery.

\section{DISCUSSION}

The incidence of aneurysm and brain tumors reported is from $0.5 \%$ to $0.7 \%(3,4,9)$ although it can be higher in patients with pituitary adenomas $(7,16)$. However, the real incidence is difficult to assess, because angiography is not always performed in patients with intracranial tumors. The coexisted aneurysm and brain tumor may not be detected until it causes symptoms. Most of the times, symptoms became apparent because of tumor growth.

The most frequent tumor associated with aneurysms is meningioma (8). In most cases, the tumor was located at the skull base, and the majority of aneurysm was detected at the internal carotid artery (ICA) and the middle cerebral artery (MCA) (2).

There have been several speculative hypotheses that have tried to explain the relationship between tumors and aneurysms. As these aneurysms used to be related to the arteries that supplies the tumor and they were found in the same hemisphere, one if the etiological factors postulated is the increased in cerebral regional blood flow. This hypothesis is supported by Tachikawa et al. (13) who reported a case of a concomitant aneurysms located on a tumor feeding artery which disappeared after tumor removal. However, this theory
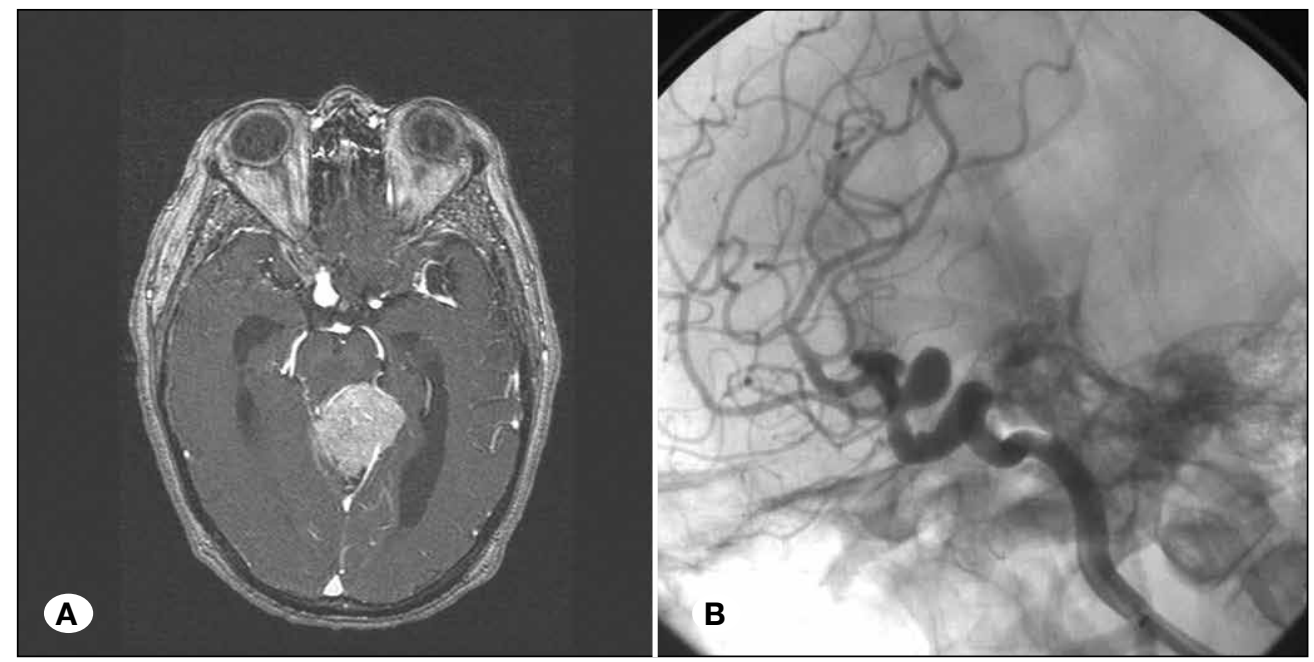

Figure 1: A) Preoperative magnetic resonance $\mathrm{T} 1$-weighted with contrast revealing a left posterior meningioma with tentorium implantation and a right posterior communicating artery aneurysm. B) Angiography image of the right posterior communicating artery aneurysm.
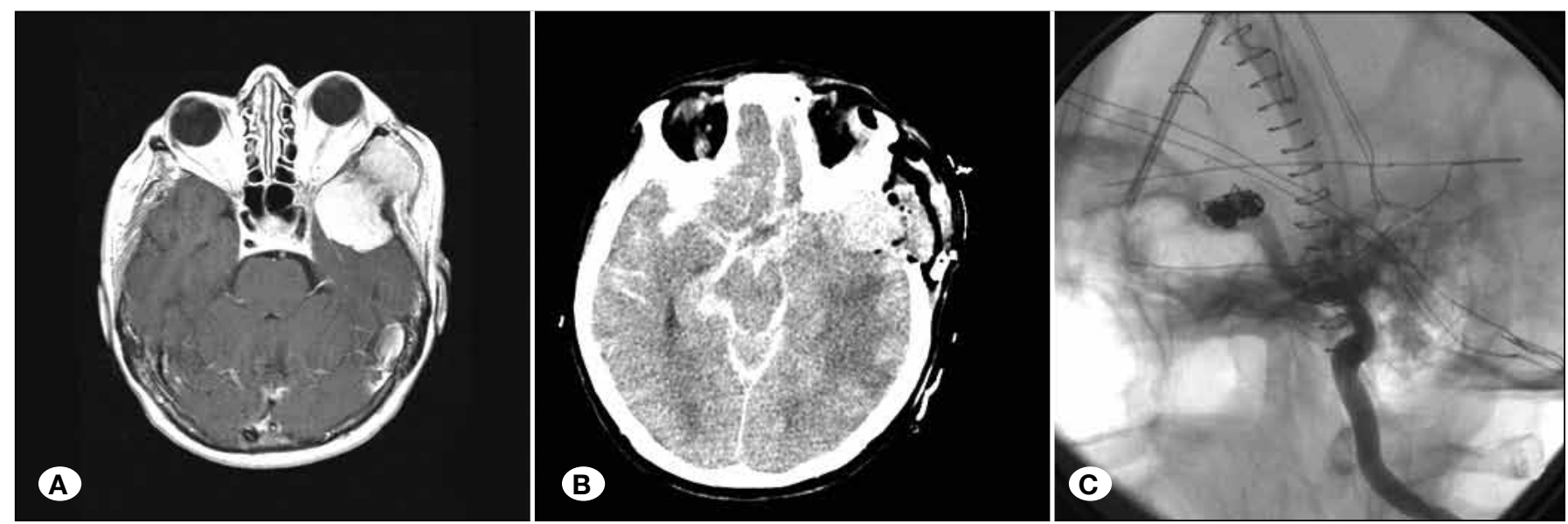

Figure 2: A) Preoperative magnetic resonance axial post-gadolinium T1-weighted sequence showing a left temporal mass. B) CT scan revealing a subarachnoid haemorrhage after craniectomy for tumor removal. C) Angiography after of paraclinoid pseudoaneurysm coiling. 
cannot explain the occurrence in different hemispheres, which seems to be coincidence. A hormonal influence, like growth factors, has been suggested in association with pituitary adenomas. Also the association between estrogen and both meningioma and aneurysms have been reported (14). Inflammatory response and meningioma adhesions can also damaged the arterial wall causing aneurysms (1). Furthermore, some mutations were found for both pathologies which show that some gene expression could be involved in development of both aneurysms and meningioma $(5,10)$.

Treatment options of both pathologies have changed, and microsurgical and endovascular techniques has reduced patients' risks (2). If the aneurysm occurs adjacent to the tumor, the first step is its occlusion and then tumor resection. If the aneurysm occurs in the other hemisphere, some authors recommend treating first the pathology that causes the symptoms (2). However, in our Case 1 the incidental aneurysm was treated first, coiling the aneurysm and then the tumor was taken with great results. In Case 2, the incidental aneurysms were not treated before the tumor, and it bleed during craniotomy. Even treating the pseudoaneurysm with endovascular coiling, it had disastrous results.

\section{CONCLUSION}

The published results suggest that the coexistence of meningioma and aneurysms in different hemispheres seems to be a coincidence. However, this rate may be underestimated. These cases show the importance of vascular study to look for feeding arteries and aneurysms. In case of aneurysms concealed by coincidental brain tumors, they should be treated before tumor resection because of the risk of rupture.

\section{REFERENCES}

1. Alnaami I: Case report: Meningioma with intra-tumoural haemorrhage secondary to ruptured distal anterior cerebral artery aneurysm. Open Neuroimag J 7:32-34, 2013

2. Fischer BR, Palkovic S, Holling M, Niederstadt T, Jeibmann A, Wassmann $\mathrm{H}$ : Coexistence of cerebral aneurysm and meningioma-pure accident? Clin Neurol Neurosurg 111(8):647-654, 2009

3. Gokalp HZ, Avman N, Ozkal E, Gokben B: Brain tumour associated with intracranial arterial aneurysm. Acta Neurochir (Wien) 53(3-4):267-273, 1980
4. Handa J, Matsuda I, Handa $\mathrm{H}$ : Association of brain tumor and intracranial aneurysms. Surg Neurol 6(1):25-29, 1976

5. Nahed BV, Bydon M, Ozturk AK, Bilguvar K, Bayrakli F, Gunel M: Genetics of intracranial aneurysms. Neurosurgery 60(2):213-225, 2007

6. Oh MC, Kim EH, Kim SH: Coexistence of intracranial aneurysm in 800 patients with surgically confirmed pituitary adenoma. J Neurosurg 116(5):942-947, 2012

7. Pant B, Arita K, Kurisu K, Tominaga A, Eguchi K, Uozumi T: Incidence of intracranial aneurysm associated with pituitary adenoma. Neurosurg Rev 20(1):13-17, 1997

8. Paraskevopoulos D, Magras I, Balogiannis I, Polyzoidis K: Anterior clinoidal meningioma coincidental with bilateral intracranial aneurysms. Hippokratia 15(4):353-355, 2011

9. Pia HW, Obrador S, Martin JG: Association of brain tumours and arterial intracranial aneurysms. Acta Neurochir (Wien) 27(3):189-204, 1972

10. Simon M, Bostrom JP, Hartmann C: Molecular genetics of meningiomas: From basic research to potential clinical applications. Neurosurgery 60(5):787-798, 2007

11. Sommet J, Schiff M, Evrard P, Blanc R, Elmaleh-Berges M: Pericallosal lipoma and middle cerebral artery aneurysm: A coincidence? Pediatr Radiol 40(8):1417-1420, 2010

12. Suslu HT, Bozbuga M: Primary brain tumors associated with cerebral aneurysm: Report of three cases. Turk Neurosurg 21(2):216-221, 2011

13. Tachikawa T, Adachi J, Nishikawa R, Matsutani M: An anterior ethmoidal artery aneurysm associated with an olfactory groove meningioma. Case illustration. J Neurosurg 97(6):1479, 2002

14. Takai K, Nishihara T, Nemoto S, Ueki K, Miyauchi H, Mishima $\mathrm{K}$, et al: Multilocular cystic lesion associated with a giant aneurysm. J Neurosurg 95(6):1081, 2001

15. Vates GE, Arthur KA, Ojemann SG, Williams F, Lawton MT: A neurocytoma and an associated lenticulostriate artery aneurysm presenting with intraventricular hemorrhage: Case report. Neurosurgery 49(3):721-725, 2001

16. Zhong Z, Sun Y, Lin D, Sun Q, Bian L: Surgical treatment of brain tumor coexisted with intracranial aneurysm-case series and review of the literature. Neurosurg Rev 36(4):645-656, 2013 